\title{
Cosmological perturbations in generalised dark Lagrangians
}

\author{
James Edholm ${ }^{a, b}$ and Jonathan Pearson ${ }^{a}$ \\ ${ }^{a}$ Consortium for Fundamental Physics, Physics Department, Lancaster University, \\ Bailrigg, Lancaster, LA1 $4 Y W$, U.K. \\ ${ }^{b}$ Centre for Particle Theory, Department of Mathematical Sciences, Durham University, \\ South Road, Durham, DH1 3LE, U.K. \\ E-mail: james.edholm@hotmail.co.uk, jon@jpoffline.com
}

AbSTRACT: We describe a new method to parameterise dark energy theories including massive gravity, elastic dark energy and tensor-metric theories. We first examine the existing framework which describes any second order Lagrangian which depends on the variation of the metric and find new constraints on the parameters. We extend the method to Lorentz violating theories which depend on the variation of the time and spatial parts of the metric separately. We show how this can describe massive gravity and elastic dark energy, while ruling out the whole class of theories where the Lagrangian depends only on the variation of the time part of the metric.

We further generalise our method to tensor-metric theories, both with and without splitting the metric into time and spatial parts. Our method extends existing physics by providing a mechanism to easily evaluate large classes of dark energy theories.

Keywords: Classical Theories of Gravity, Space-Time Symmetries, Spacetime Singularities

ARXIV EPRINT: 1810.06582 


\section{Contents}

1 Introduction 1

1.1 Decomposition of the metric 2

$\begin{array}{lll}2 & \mathcal{L}_{\{2\}}\left(\delta_{L} g_{\mu \nu}\right) & 3\end{array}$

2.1 Contractions of the Eulerian change in the energy-momentum tensor 3

2.2 Perturbed fluid variables 4

2.3 Invariance under time reparameterisation 5

$3 \mathcal{L}_{\{2\}}\left(\delta_{L} u_{\mu}, \delta_{L} \gamma_{\mu \nu}\right) \quad 6$

3.1 Decomposition of the $X, Y$ and $Q$ tensors 7

3.2 The specific $W$ tensor for $\mathcal{L}\left(\delta_{L} u_{\mu}, \delta_{L} \gamma_{\mu \nu}\right) \quad 7$

3.3 Invariance under time reparameterisation 8

$\begin{array}{lll}3.3 .1 & \mathcal{L}_{\{2\}}\left(\delta_{L} u_{\mu}\right) & 8\end{array}$

$\begin{array}{lll}3.3 .2 & \mathcal{L}_{\{2\}}\left(\delta_{L} \gamma_{\mu \nu}\right) & 9\end{array}$

3.3.3 Summary of $\mathcal{L}_{\{2\}}\left(\delta_{L} u_{\mu}, \delta_{L} \gamma_{\mu \nu}\right) \quad 9$

3.4 Comparison with elastic dark energy theories 9

4 Tensor-metric theories $\quad 10$

$\begin{array}{ll}4.1 \text { Equations of motion } & 11\end{array}$

5 No preferred direction in the coupling tensors $\quad 12$

5.1 Preferred direction 12

$\begin{array}{ll}5.2 \text { The perturbed fluid variables } & 12\end{array}$

6 Choosing a form for $f_{\mu \nu} \quad 13$

$\begin{array}{lll}\text { 6.1 Conformal and disformal choices } & 13\end{array}$

$\begin{array}{lll}6.2 & \text { Flat reference metric } & 13\end{array}$

$\begin{array}{lll}7 & \text { Summary of results } & 14\end{array}$

$\begin{array}{ll}\text { A Evolution of } w & 14\end{array}$

$\begin{array}{ll}\text { A.1 Sound speed } & 15\end{array}$

\section{Introduction}

The discovery of the accelerated expansion of the universe [1-8] prompted many attempts to explain the phenomenon, caused by an unknown "dark energy", using modifications to Einstein's theory of General Relativity [9]. These theories generally started with a specific dark Lagrangian which was then investigated to test its compatibility with both dark 
energy and other cosmological and solar system observations. Here we follow the method of [9-17] and look at a class of dark Lagrangians, so that future experimental results can immediately rule out a whole swathe of models without the need for further calculation.

We use the formalism introduced in [10-14] which draws on the "Post Parameterised Friedmann" (PPF) approach described in [9, 15-17]. In these papers the Einstein-Hilbert action is modified with a dark Lagrangian such that the modified action is written as

$$
S=\int d^{4} x \sqrt{-g}\left[\frac{R}{16 \pi G}-\mathcal{L}_{\text {matter }}-\mathcal{L}_{d}\right]
$$

and equations of state for dark sector perturbations are found for the entropy perturbation and the anisotropic stress. The recent observation of a neutron star merger by LIGO [18] severely constrained the speed of gravitational waves, and seemingly placed strict restrictions on many modified gravity theories. However, it was suggested in [19] that although these theories predict the speed of gravitational waves to be different from that of light at low energies, this may not necessarily be the case at the high energies seen in neutron star mergers, due to the unknown UV completions of these theories.

We find the perturbed fluid variables when $\mathcal{L}_{\{2\}}=\mathcal{L}_{\{2\}}\left(\delta_{L} g_{\mu \nu}\right)$, in a similar way to [12]. We then impose invariance under time reparameterisation and find the equations of motion. We also find evolution equations for the equation of state parameter $w$ and the elastic bulk modulus. We find restrictions on $w$ such that we can have a realistic (positive and subluminal) sound speed. We find the perturbed fluid variables when we have imposed spatial invariance, and we find the conditions under which the entropy is gauge invariant.

In this paper our main focus is on generalising all of these calculations for the case where $\mathcal{L}_{\{2\}}$ is a function of the change in the time-like and spatial parts of the metric separately, not necessarily packaged together as the spacetime metric.

Elastic dark energy (EDE) discussed in $[12,20]$ is a development of Carter-Quintana relativistic elasticity theory [21-25]. These models describe the universe as a solid with certain parameters which can be found by observations. By imposing time translational invariance but not spatial invariance, we fulfill the conditions necessary for EDE.

As we are adding a dark energy term to the Einstein-Hilbert action (1.1), we can

decompose the stress energy tensor as $T_{\mu \nu}=T_{\mu \nu}^{\text {matter }}+T_{\mu \nu}^{\text {radiation }}+T_{\mu \nu}^{\text {dark }}$, i.e. we have added a source term to the standard model stress energy tensor.

\subsection{Decomposition of the metric}

We impose spatial isotropy by foliating the four-dimensional (4D) space-time, as in [12, 26], by three-dimensional (3D) sheets with a time-like unit vector, $u_{\mu}$ which is everywhere orthogonal to the sheets. The $4 \mathrm{D}$ spacetime has metric $g_{\mu \nu}$, and the $3 \mathrm{D}$ sheets have spatial metric $\gamma_{\mu \nu}=\gamma_{(\mu \nu)}$. The $(3+1)$ decomposition of the $4 \mathrm{D}$ metric is

$$
g_{\mu \nu}=\gamma_{\mu \nu}-u_{\mu} u_{\nu}
$$

where $u_{\mu}$ and $\gamma_{\mu \nu}$ are subject to the orthogonality and normality conditions:

$$
u^{\mu} \gamma_{\mu \nu}=0, \quad u^{\mu} u_{\mu}=-1
$$


Hence we can write the dark term in the stress-energy tensor in an isotropic spacetime as

$$
T_{\mu \nu}=\rho u_{\mu} u_{\nu}+P \gamma_{\mu \nu}
$$

where $\rho$ is the density of the universe due to this dark term and $P$ is the pressure. The pressure and density are related by $P=w \rho$, where $w$ is the equation of state parameter. From now on, when we write $T_{\mu \nu}$, this will refer to the dark stress-energy tensor rather than the overall energy momentum tensor.

\section{$2 \mathcal{L}_{\{2\}}\left(\delta_{L} g_{\mu \nu}\right)$}

We will first look at the case $\mathcal{L}_{\{2\}}\left(\delta_{L} g_{\mu \nu}\right)$ before generalising to more generic Lagrangians. For any Lagrangian which is a function only of perturbations of the metric and no derivatives thereof, i.e. $\mathcal{L}_{\{2\}}=\mathcal{L}_{\{2\}}\left(\delta_{L} g_{\mu \nu}\right)$, then from [12], the most general quadratic Lagrangian for dark sector perturbations is

$$
\mathcal{L}_{\{2\}}=\frac{1}{8} W^{\mu \nu \alpha \beta} \delta_{L} g_{\mu \nu} \delta_{L} g_{\alpha \beta},
$$

where $\delta_{L} g_{\mu \nu}$ is the metric fluctuation under a perturbation and $W^{\mu \nu \alpha \beta}$ can be thought of as a mass term for the perturbation, not to be confused with the Weyl tensor. From [13], we can use the symmetries of the $W$ tensor:

$$
W_{\mu \nu \alpha \beta}=W_{(\mu \nu)(\alpha \beta)}=W_{\alpha \beta \mu \nu},
$$

to obtain the most general possible $W_{\alpha \beta \mu \nu}$ :

$$
\begin{aligned}
W_{\mu \nu \alpha \beta}= & A_{W} u_{\mu} u_{\nu} u_{\alpha} u_{\beta}+B_{W}\left(u_{\mu} u_{\nu} \gamma_{\alpha \beta}+\gamma_{\mu \nu} u_{\alpha} u_{\beta}\right) \\
& +C_{W} u_{(\mu} \gamma_{\nu)(\alpha} u_{\beta)}+D_{W} \gamma_{\mu \nu} \gamma_{\alpha \beta}+E_{W} \gamma_{\mu(\alpha} \gamma_{\beta) \nu} .
\end{aligned}
$$

\subsection{Contractions of the Eulerian change in the energy-momentum tensor}

The deformation vector, which represents possible coordinate changes $[14],{ }^{1}$ has both timelike parts and space-like parts

$$
\xi_{\mu}=-\chi u_{\mu}+m_{\mu}
$$

We can find the various contractions of $\delta_{E} T^{\mu}{ }_{\nu}$ by inserting the derivatives of the deformation vector. We use two different variational operators: $\delta_{L}$ and $\delta_{E}$, which are linked via the Lie derivative along the diffeomorphism-generating vector $\xi^{\mu}$ via

$$
\delta_{L}=\delta_{E}+L_{\xi}
$$

where $\delta_{L}$ is the Lagrangian perturbation (in co-moving coordinates) and $\delta_{E}$ is the Eulerian perturbation (points are fixed in spacetime). $L_{\xi}$ is the Lie derivative in the direction of $\xi^{\mu}$, a vector field representing possible coordinate transformations, so that the Lie derivative acting on a given symmetric tensor field is $L_{\xi} X_{\mu \nu}=\xi^{\alpha} \nabla_{\alpha} X_{\mu \nu}+2 X_{\alpha(\mu} \nabla_{\alpha} \xi^{\nu)}$. Note that

\footnotetext{
${ }^{1}$ The equations of motion of General Relativity are independent of $\xi_{\mu}$, but this is not necessarily true for more general actions. We can decompose $\delta_{L} g_{\mu \nu}$ as $\delta_{L} g_{\mu \nu}=h_{\mu \nu}+2 \nabla_{(\mu} \xi_{\nu)}$ [14].
} 
$L_{\xi} g_{\mu \nu}=2 \nabla_{(\mu} \xi_{\nu)}$. We are interested in the perturbed fluid equations, which are derived from the Lagrangian for perturbations, denoted as $\mathcal{L}_{(2)}$. In order to find the perturbed fluid variables, we first need to find the Eulerian change in the stress-energy tensor, $\delta_{E} T^{\mu \nu}$. We will work in the synchronous gauge, where perturbations to the metric have spatial components only

$$
\delta_{E} g_{\mu \nu}=\gamma_{\mu}^{\alpha} \gamma_{\nu}^{\beta} h_{\alpha \beta}
$$

Working in the synchronous gauge (2.6), we vary (2.1) to obtain the contractions of the stress energy tensor $[12,14]$

$$
\begin{aligned}
u_{\mu} u^{\nu} \delta_{E} T_{\nu}^{\mu}= & \left(\dot{\rho}+K\left(B_{W}+\rho\right)\right) \chi-\left(B_{W}+\rho\right)\left(\frac{1}{2} \hat{h}+\nabla_{\alpha} m^{\alpha}\right)-\left(\rho+A_{W}\right) \dot{\chi} \\
u^{\nu} \gamma_{\mu}^{\sigma} \delta_{E} T_{\nu}^{\mu}= & \left(\frac{1}{4} C_{W}+P\right) \bar{\nabla}^{\sigma} \chi-\frac{1}{4} C_{W} K^{\sigma \beta} m_{\beta}+\left(\frac{1}{4} C_{W}-\rho\right) \dot{m}^{\sigma}+\rho K_{\alpha}^{\sigma} m^{\alpha} \\
\gamma_{\mu}^{\nu} \delta_{E} T_{\nu}^{\mu}= & -\frac{1}{2}\left(\gamma^{\alpha \beta}\left(3 D_{W}+E_{W}+P\right)+3 u^{\alpha} u^{\beta}\left(B_{W}-P\right)\right) \delta_{E} g_{\alpha \beta} \\
& -\left[3 u^{\alpha} u^{\beta}\left(B_{W}-P\right)+\gamma^{\alpha \beta}\left(3 D_{W}+E_{W}+P\right)\right] \nabla_{\alpha} \xi_{\beta}+3 \chi \dot{P} \\
\gamma_{\mu}^{\sigma} \gamma_{\rho}^{\nu} \delta_{E} T_{\nu}^{\mu}= & \chi \gamma_{\rho}^{\sigma} \dot{P}-\frac{1}{2}\left(\left(D_{W}+P\right) \gamma_{\rho}^{\sigma} \hat{h}+\left(E_{W}-2 P\right) \hat{h}_{\rho}^{\sigma}\right) \\
& +\gamma_{\rho}^{\sigma}\left[\left(P-A_{W}\right) \dot{\chi}-\left(D_{W}+P\right)\left(\bar{\nabla}_{\alpha} m^{\alpha}-\chi K\right)\right] \\
& +\left(2 P-F_{W}\right)\left[\bar{\nabla}^{(\sigma} m_{\rho)}-m_{\beta} K^{\beta(\sigma} u_{\rho)}-\chi K_{\rho}^{\sigma}\right]
\end{aligned}
$$

where we have defined "time" and "space" differentiation as the derivative operator projected along the time and space directions

$$
\dot{\psi} \equiv u^{\mu} \nabla_{\mu} \psi, \quad \bar{\nabla}_{\mu} \psi \equiv \gamma_{\mu}^{\nu} \nabla_{\nu} \psi
$$

and where $K=3 H$, (where $H$ is the Hubble parameter) is the trace of the extrinsic curvature tensor $K_{\mu \nu} \equiv \nabla_{\mu} u_{\nu}$, which satisfies $K_{\mu \nu}=K_{(\mu \nu)}$ and $u^{\mu} K_{\mu \nu}=0$.

\section{$2.2 \quad$ Perturbed fluid variables}

The components of the perturbed energy-momentum tensor $T^{\mu}{ }_{\nu}$ are written as

$$
\delta_{E} T^{\mu}{ }_{\nu}=\delta \rho u^{\mu} u_{\nu}+2(\rho+P) v^{(\mu} u_{\nu)}+\delta P \gamma^{\mu}{ }_{\nu}+P \Pi^{\mu}{ }_{\nu},
$$

where $v^{\mu}$ is the perturbed dark sector velocity and $P \Pi^{\mu}{ }_{\nu}$ is the anisotropic part of the stress tensor, which is orthogonal and symmetric. We have dropped the subscript $E$ on the variation of the density and pressure. We can now compare (2.9) and the contractions of the perturbed stress energy tensor (2.7), to obtain the perturbed fluid variables in terms 
of the deformation vector $(2.4)$, as described in $[12,14]$

$$
\begin{aligned}
\delta \rho= & \left(\dot{\rho}+K\left(B_{W}+\rho\right)\right) \chi-\left(\rho+A_{W}\right) \dot{\chi}-\left(B_{W}+\rho\right)\left(\frac{1}{2} \hat{h}+\bar{\nabla}_{\alpha} m^{\alpha}\right), \\
\delta P= & \left(P-B_{W}\right) \dot{\chi}-\frac{1}{3}\left(3 D_{W}+E_{W}+P\right)\left(\frac{1}{2} \hat{h}+\bar{\nabla}_{\alpha} m^{\alpha}\right) \\
& +\left[\frac{1}{3}\left(3 D_{W}+E_{W}+P\right) K+\dot{P}\right] \chi \\
(\rho+P) v^{\sigma}= & \left(\rho-\frac{C_{W}}{4}\right) \dot{\bar{m}}^{\sigma}-\left(P+\frac{C_{W}}{4}\right) \bar{\nabla}^{\sigma} \chi \\
P \Pi_{\rho}^{\sigma}= & \left(2 P-E_{W}\right)\left[\frac{1}{2} \hat{h}_{\rho}^{\sigma}+\bar{\nabla}^{(\sigma} m_{\rho)}-\frac{1}{3} \gamma_{\rho}^{\sigma}\left(\frac{1}{2} \hat{h}+\bar{\nabla}^{\alpha} m_{\alpha}\right)\right]
\end{aligned}
$$

where we have defined $P \Pi^{\mu}{ }_{\nu}=\left(\gamma^{\mu}{ }_{\beta} \gamma^{\alpha}{ }_{\nu}-\frac{1}{3} \gamma^{\alpha}{ }_{\beta} \gamma^{\mu}{ }_{\nu}\right) \delta_{E} T^{\beta}{ }_{\alpha}$.

\subsection{Invariance under time reparameterisation}

We now want to discover what constraints invariance under changes in time imposes. This is helpful for $[12,20]$ because applying time translational reparameterisation invariance but not spatial reparameterisation invariance leads to elastic dark energy. Hence we set the coefficients of $\chi, \dot{\chi}$ and $\bar{\nabla}^{\sigma} \chi$ in (2.10) to zero and therefore obtain

$$
\begin{aligned}
\dot{\rho}+3 H(P+\rho) & =0, \\
\dot{P}+\left(P+3 D_{W}+E_{W}\right) H & =0 .
\end{aligned}
$$

This gives the conservation equation and an equation for the evolution of pressure with time. We can rewrite the perturbed fluid variables (2.10) as

$$
\begin{aligned}
\delta \rho & =-(P+\rho)\left(\frac{1}{2} \hat{h}+\bar{\nabla}_{\alpha} \xi^{\alpha}\right), \\
\delta P & =-\beta\left(\frac{1}{2} \hat{h}+\bar{\nabla}_{\alpha} \xi^{\alpha}\right), \\
v^{\sigma} & =\dot{\xi}^{\sigma}, \\
P \Pi_{\rho}^{\sigma} & =2 \mu\left[\frac{1}{2} \hat{h}_{\rho}^{\sigma}+\bar{\nabla}^{(\sigma} \xi_{\rho)}-\frac{1}{3} \gamma_{\rho}^{\sigma}\left(\frac{1}{2} \hat{h}+\bar{\nabla}^{\alpha} \xi_{\alpha}\right)\right],
\end{aligned}
$$

and the pressure evolution as

$$
\dot{P}+3 \beta H=0,
$$

where we have defined

$$
\begin{aligned}
\beta & =\frac{1}{3} P+D_{W}+\frac{1}{3} E_{W}, \\
\mu & =P-\frac{1}{2} E_{W},
\end{aligned}
$$

as parameters that can be determined by experiment. $\beta$ and $\mu$ correspond to the elastic bulk modulus and the elastic shear modulus, respectively $[12,20]$. 
So far, we have summarised previous work. However, as an aside we note that it is possible to place constraints on the parameters using observational data. In [27], it was noted that it might be possible to place constraints on the parameters of the elastic dark energy model, referred to as Time Diffeomorphism Invariant (TDI) models in their paper. The authors used observational data on cosmic shear and CMB lensing which would give constraints for any given value of $w$, unless $w \approx-1$, as a wide. Unfortunately, recent Planck data has shown that $w \approx-1$, so these constraint do not apply.

In appendix A, using 2018 Planck data [28], we find a constraint of $-0.004<\beta<$ 0.106 if we assume that the equation of state parameter $w$ of the Lagrangian (2.1), where $P=w \rho$, is constant. If we assume that $w \neq-1$ exactly, then we also find constraints of $-0.0477 \leq \hat{\mu} \leq 0.0599$, where we have defined $\hat{\mu}=\mu / \rho$. However,the assumption that we do not have a phantom equation of state, i.e. we require $w \geq-1$, gives $0 \leq \hat{\mu} \leq 0.0599$.

\section{$3 \mathcal{L}_{\{2\}}\left(\delta_{L} u_{\mu}, \delta_{L} \gamma_{\mu \nu}\right)$}

In the previous section, we summarised the derivation of the perturbed fluid variables for theories where the second variation of the Lagrangian depends only on the change in the metric [12] and found new constraints on the values of the elasticity and rigidity parameters. We now move on to extend this work to more general Lagrangians.

If we take the second variation of the Lagrangian as a function only of the change in the time and spatial parts of the metric separately

$$
\mathcal{L}_{\{2\}}=\mathcal{L}_{\{2\}}\left(\delta_{L} u_{\mu}, \delta_{L} \gamma_{\mu \nu}\right)
$$

then the most general possible quadratic Lagrangian which is a function of $\delta_{L} u_{\mu}$ and $\delta_{L} \gamma_{\mu \nu}$ is

$$
\mathcal{L}_{\{2\}}=\frac{1}{8} X^{\mu \nu \alpha \beta} \delta_{L} \gamma_{\mu \nu} \delta_{L} \gamma_{\alpha \beta}+\frac{1}{8} Y^{\mu \nu} \delta_{L} u_{\mu} \delta_{L} u_{\nu}+\frac{1}{4} Q^{\mu \nu \alpha} \delta_{L} u_{\mu} \delta_{L} \gamma_{\nu \alpha}
$$

where

$$
X^{\mu \nu \alpha \beta}=X^{(\mu \nu)(\alpha \beta)}=X^{\alpha \beta \mu \nu}, \quad Y^{\mu \nu}=Y^{(\mu \nu)}, \quad Q^{\mu \nu \alpha}=Q^{\mu(\nu \alpha)} .
$$

Using the identities [20]

$$
\begin{aligned}
\delta_{L} u^{\mu} & =\frac{1}{2} u^{\mu} u^{\alpha} u^{\beta} \delta_{L} g_{\alpha \beta}, \\
\delta_{L} \gamma_{\mu \nu} & =\delta_{L} g_{\mu \nu}+2 u_{(\mu}\left(\gamma^{\alpha}{ }_{\nu)}-\frac{1}{2} u_{\nu)} u^{\alpha}\right) u^{\beta} \delta_{L} g_{\alpha \beta},
\end{aligned}
$$

it is possible to write $(3.2)$ as

$$
\mathcal{L}_{\{2\}}=\left(W_{X}^{\mu \nu \alpha \beta}+W_{Y}^{\mu \nu \alpha \beta}+W_{Z}^{\mu \nu \alpha \beta}\right) \delta_{L} g_{\alpha \beta} \delta_{L} g_{\mu \nu},
$$


where upon comparing (3.2) with (2.1), we can see that the components of $W^{\sigma \rho \phi \lambda}=$ $W_{X}^{\sigma \rho \phi \lambda}+W_{Y}^{\sigma \rho \phi \lambda}+W_{Q}^{\sigma \rho \phi \lambda}$ are

$$
\begin{aligned}
& W_{X}^{\sigma \rho \phi \lambda}=\frac{1}{2} X^{\mu \nu \alpha \beta}\left[2\left(\delta^{(\sigma}{ }_{\mu} \delta^{\rho)}{ }_{\nu} \delta^{(\phi}{ }_{\alpha} \delta^{\lambda)}{ }_{\beta}+\delta^{\left(\phi_{\mu}\right.}{ }^{\lambda}{ }^{\lambda)}{ }_{\nu} \delta^{(\sigma}{ }_{\alpha} \delta^{\rho)}{ }_{\beta}\right)+4 u_{(\mu} H^{\sigma \rho}{ }_{\nu)}\right.
\end{aligned}
$$

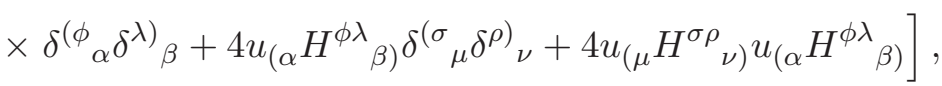

$$
\begin{aligned}
& W_{Y}^{\sigma \rho \phi \lambda}=\frac{1}{4} Y^{\mu \nu}\left[H^{\phi \lambda}{ }_{\mu} H_{\nu}^{\sigma \rho}+H_{\nu}^{\phi \lambda} H_{\mu}^{\sigma \rho}\right] \text {, } \\
& W_{Q}^{\sigma \rho \phi \lambda}=2 Q^{\mu \nu \alpha}\left(\delta^{(\sigma}{ }_{\mu} \delta^{\rho)}{ }_{\nu}+u_{(\mu} H^{\sigma \rho}{ }_{\nu}\right) H^{\phi \lambda}{ }_{\alpha},
\end{aligned}
$$

where we have defined

$$
H_{\mu}^{\alpha \beta} \equiv\left(\gamma_{\mu}^{(\alpha}-\frac{1}{2} u_{\mu} u^{(\alpha}\right) u^{\beta)}
$$

Eq. (3.5) is a very important result - we can rewrite any Lagrangian dependent on the variation of the spatial part of the metric and the time-like part of the metric separately into one dependent on only the variation of the full metric.

In the rest of this section, we will explore how we can use the method of section 2 to find the perturbations of a Lagrangian of the form (2) without any new calculations.

\subsection{Decomposition of the $X, Y$ and $Q$ tensors}

The most general tensors that satisfy the necessary symmetries (3.3) are:

$$
\begin{aligned}
X^{\mu \nu \alpha \beta}= & A_{X} u^{\mu} u^{\nu} u^{\alpha} u^{\beta}+B_{X}\left(u^{\mu} u^{\nu} \gamma^{\alpha \beta}+\gamma^{\mu \nu} u^{\alpha} u^{\beta}\right)+4 C_{X} u^{(\mu} \gamma^{\nu)(\alpha} u^{\beta)}, \\
& +D_{X} \gamma^{\mu \nu} \gamma^{\alpha \beta}+\frac{1}{2} E_{X} \gamma^{\mu(\alpha} \gamma^{\beta) \nu}, \\
Y^{\mu \nu}= & A_{Y} u^{\mu} u^{\nu}+B_{Y} \gamma^{\mu \nu} \\
Q^{\mu \nu \alpha}= & A_{Q} u^{\mu} u^{\nu} u^{\alpha}+B_{Q} u^{\mu} \gamma^{\nu \alpha}+2 C_{Q} u^{(\nu} \gamma^{\alpha) \mu} .
\end{aligned}
$$

\subsection{The specific $W$ tensor for $\mathcal{L}\left(\delta_{L} u_{\mu}, \delta_{L} \gamma_{\mu \nu}\right)$}

Collecting like terms, we obtain

$$
\begin{aligned}
W^{\sigma \rho \phi \lambda}= & W_{X}^{\sigma \rho \phi \lambda}+W_{Y}^{\sigma \rho \phi \lambda}+W_{Q}^{\sigma \rho \phi \lambda} \\
= & \left(\frac{1}{2} A_{X}+\frac{1}{8} A_{Y}+\frac{1}{2} A_{Q}\right) u^{\sigma} u^{\rho} u^{\phi} u^{\lambda}+B_{X}\left(u^{\sigma} u^{\rho} \gamma^{\phi \lambda}+\gamma^{\sigma \rho} u^{\phi} u^{\lambda}\right) \\
& +\left(8 C_{X}+\frac{1}{2} B_{Y}+B_{Q}+2 C_{Q}\right) u^{(\sigma} \gamma^{\rho)(\phi} u^{\lambda)}+2 D_{X} \gamma^{\sigma \rho} \gamma^{\phi \lambda}+E_{X} \gamma^{\phi(\sigma} \gamma^{\rho) \lambda} .
\end{aligned}
$$

Comparing to (2.3), we find

$$
\begin{aligned}
A_{W} & =\frac{1}{2} A_{X}+\frac{1}{8} A_{Y}+\frac{1}{2} A_{Q}, \\
B_{W} & =B_{X}, \\
C_{W} & =8 C_{X}+\frac{1}{2} B_{Y}+B_{Q}+2 C_{Q}, \\
D_{W} & =D_{X}, \\
E_{W} & =E_{X} .
\end{aligned}
$$




\subsection{Invariance under time reparameterisation}

We repeat our calculations from earlier, where we fixed our equations to be the same under a change in time and we obtained (2.13).

Plugging in the values from (3.10), we find an equation for the evolution of the pressure

$$
\dot{P}=-\left(P+3 D_{X}+E_{X}\right) H=-3 \beta_{g} H,
$$

where $\beta_{g} \equiv \frac{1}{3}\left(P+3 D_{X}+E_{X}\right)$. We also find that the coefficients of the $W$ tensor become

$$
\begin{aligned}
A_{W} & =\frac{1}{2} A_{X}+\frac{1}{8} A_{Y}+\frac{1}{2} A_{Q}=\rho, \\
B_{W} & =B_{X}=P, \\
C_{W} & =8 C_{X}+\frac{1}{2} B_{Y}+B_{Q}+2 C_{Q}=-P, \\
D_{W} & =D_{X}, \\
E_{W} & =E_{X} .
\end{aligned}
$$

Next we will examine the specific cases where the Lagrangian depends only on the variation of either the time or the spatial part of the metric.

\subsection{1 $\mathcal{L}_{\{2\}}\left(\delta_{L} u_{\mu}\right)$}

First, if we make $\mathcal{L}_{\{2\}}$ a function of $\delta_{L} u_{\mu}$ only, i.e. $\mathcal{L}_{\{2\}}$ is dependent only on the change in the time part of the metric, we obtain $W_{X}=W_{Q}=0$, in which case $\beta=P=0$, and using $(2.14 \mathrm{~b})$ we can then rewrite the perturbed fluid variables from $(2.12)$

$$
\begin{aligned}
\delta \rho & =-\rho\left(\frac{1}{2} \hat{h}+\bar{\nabla}_{\alpha} \xi^{\alpha}\right), \\
\delta P & =0 \\
(1+w) v^{\sigma} & =\dot{\xi}^{\sigma}, \\
P \Pi_{\rho}^{\sigma} & =0 .
\end{aligned}
$$

We find that the equation of state parameter is

$$
w=\dot{w}=0 .
$$

Using constraints from Planck [28] which show $w \approx-1$, we can therefore rule out this case. While this case might intuitively seem unlikely, ${ }^{2}$ we have completely ruled it out without having to examine any specific model. This shows the power of our parameterisation method.

\footnotetext{
${ }^{2}$ The lack of spatial dependence means that the dark energy Lagrangian is described by a pressureless fluid.
} 


\subsection{2 $\mathcal{L}_{\{2\}}\left(\delta_{L} \gamma_{\mu \nu}\right)$}

If we make $\mathcal{L}$ a function of $\delta_{L} \gamma_{\mu \nu}$ only, i.e. $\mathcal{L}$ is dependent only on the change in the spatial part of the metric, we get $W_{Y}=W_{Q}=0$, which means

$$
\begin{aligned}
A_{W} & =\frac{1}{2} A_{X}=\rho, \\
B_{W} & =B_{X}=P, \\
C_{W} & =8 C_{X}=-P, \\
D_{W} & =D_{X}, \\
E_{W} & =E_{X},
\end{aligned}
$$

and

$$
\begin{aligned}
\dot{P} & =-\left(P+3 D_{X}+E_{X}\right) H \\
& \equiv-3 \beta_{\gamma} H .
\end{aligned}
$$

where we have defined $\beta_{\gamma}=\frac{1}{3}\left(P+3 D_{X}+E_{X}\right)$. For $\mathcal{L}_{\{2\}}\left(\delta_{L} \gamma_{\mu \nu}\right)$ the evolution equation for $w$ (A.2) remains the same but with $\beta$ now depending on $D_{X}$ and $E_{X}$ rather than $D$ and $F$, which lead to the same result if we use (3.15).

\subsubsection{Summary of $\mathcal{L}_{\{2\}}\left(\delta_{L} u_{\mu}, \delta_{L} \gamma_{\mu \nu}\right)$}

The analysis of section 3 shows that the effect of the metric split is simply to change the coefficients as shown in (3.10).

If we have mandated time reparameterisation invariance by decoupling $\chi$, then the only relevant contribution to $\beta$ and $\mu$ come from the spatial part of the metric $\gamma_{\mu \nu}$, i.e. only the first term in (3.2) has any effect on the system.

\subsection{Comparison with elastic dark energy theories}

We can compare to [20] to find the properties of the dark energy material, as this Lagrangian can be described using elastic dark energy.

We perform a scalar-vector-tensor (SVT) decomposition, where we decompose the perturbation to the metric $h_{i j}$ as [29]

$$
\frac{1}{2} h_{i j}=H_{L}^{S} Q_{i j}^{S}+H_{T}^{S} Q_{i j}^{S}+H^{V} Q_{i j}^{V}+H^{T} Q_{i j}^{T}
$$

where one can decompose a spatial tensor field as

$$
\eta^{i j} \nabla_{i} \nabla_{j} Q^{S, V, T}=-k^{2} Q^{S, V, T},
$$

scalars can be constructed from vectors and tensors as [30]

$$
\nabla_{i} Q^{S}=-k Q_{i}^{S}, \quad \nabla_{i} \nabla_{j} Q^{S}+\frac{1}{3} k^{2} \eta_{i j} Q^{S}=Q_{i j}^{S},
$$

and vectors can be constructed from tensors as

$$
\nabla_{(i} Q_{j)}^{V}=-k Q_{i j}^{V}
$$


with the requirement that $Q_{i}^{V \mid i}=Q_{i j}^{T \mid i}=Q_{i}^{T i}=0$. We now find the entropy perturbation, which is defined by

$$
w \Gamma=\left(\frac{\delta P}{\delta \rho}-\frac{d P}{d \rho}\right) \delta
$$

The entropy perturbation and scalar anisotropic stress are given by

$$
\begin{aligned}
w \Gamma & =0, \\
w \Pi^{S} & =-2 \frac{\mu}{\rho+P}[\delta-3(1+w) \eta],
\end{aligned}
$$

where we have defined $\delta=\frac{\delta \rho}{\rho}$, and $\eta=-\left(H_{L}^{S}+\frac{1}{3} H_{T}^{S}\right)$. This is the same result as [20] finds when looking at elastic mediums.

\section{Tensor-metric theories}

So far, we have looked at theories where $\mathcal{L}_{\{2\}}=\mathcal{L}_{\{2\}}\left(\delta g_{\mu \nu}, \delta f_{\mu \nu}\right)$. In this section, we now apply our analysis to theories where the dark Lagrangian is a function of both the variation of the metric $g_{\mu \nu}$ and of an unspecified non-dynamical symmetric rank-2 tensor $f_{\mu \nu}[31,32] .^{3}$

If $\mathcal{L}_{\{2\}}=\mathcal{L}_{\{2\}}\left(\delta g_{\mu \nu}, \delta f_{\mu \nu}\right)$, then the most general quadratic Lagrangian we can have is

$$
\mathcal{L}_{\{2\}}=\frac{1}{8} A^{\mu \nu \alpha \beta} \delta_{L} g_{\mu \nu} \delta_{L} g_{\alpha \beta}+\frac{1}{4} B^{\mu \nu \alpha \beta} \delta_{L} g_{\mu \nu} \delta_{L} f_{\alpha \beta}+\frac{1}{8} C^{\mu \nu \alpha \beta} \delta_{L} f_{\mu \nu} \delta_{L} f_{\alpha \beta} .
$$

The tensors obey the following symmetries

$$
A^{\mu \nu \alpha \beta}=A^{(\mu \nu)(\alpha \beta)}=A^{\alpha \beta \mu \nu}, \quad B^{\mu \nu \alpha \beta}=B^{(\mu \nu)(\alpha \beta)}, \quad C^{\mu \nu \alpha \beta}=C^{(\alpha \beta)(\mu \nu)} .
$$

The decomposition of these tensors, called "coupling tensors" because they prescribe how the fields combine in the Lagrangian, is

$$
\begin{aligned}
A^{\mu \nu \alpha \beta}= & A_{X} u^{\mu} u^{\nu} u^{\alpha} u^{\beta}+B_{X}\left(u^{\mu} u^{\nu} \gamma^{\alpha \beta}+\gamma^{\mu \nu} u^{\alpha} u^{\beta}\right)+4 C_{X} u^{(\mu} \gamma^{\nu)(\alpha} u^{\beta)} \\
& +D_{X} \gamma^{\mu \nu} \gamma^{\alpha \beta}+2 E_{X} \gamma^{\mu(\alpha} \gamma^{\beta) \nu}, \\
B^{\mu \nu \alpha \beta}= & A_{Y} u^{\mu} u^{\nu} u^{\alpha} u^{\beta}+B_{Y} u^{\mu} u^{\nu} \gamma^{\alpha \beta}+4 C_{Y} u^{(\mu} \gamma^{\nu)(\alpha} u^{\beta)} \\
& +D_{Y} \gamma^{\mu \nu} \gamma^{\alpha \beta}+2 E_{Y} \gamma^{\mu(\alpha} \gamma^{\beta) \nu}+F_{Y} \gamma^{\mu \nu} u^{\alpha} u^{\beta}, \\
C^{\mu \nu \alpha \beta}= & A_{Z} u^{\mu} u^{\nu} u^{\alpha} u^{\beta}+B_{Z}\left(u^{\mu} u^{\nu} \gamma^{\alpha \beta}+\gamma^{\mu \nu} u^{\alpha} u^{\beta}\right)+4 C_{Z} u^{(\mu} \gamma^{\nu)(\alpha} u^{\beta)} \\
& +D_{Z} \gamma^{\mu \nu} \gamma^{\alpha \beta}+2 E_{Z} \gamma^{\mu(\alpha} \gamma^{\beta) \nu} .
\end{aligned}
$$

\footnotetext{
${ }^{3}$ It should be noted that at this point there are no derivatives of $f_{\mu \nu}$ in the action and therefore no kinetic term for $f_{\mu \nu}$. We could choose a form of $f_{\mu \nu}$ that includes derivatives of a vector field or a scalar, and therefore generates a kinetic term.
} 


\subsection{Equations of motion}

We use (4.3) to find

$$
\begin{aligned}
\delta_{E} T^{\mu}{ }_{\nu}= & -\frac{1}{2}\left(B_{X} u^{\mu} u_{\nu}+D_{X} \gamma^{\mu}{ }_{\nu}+T^{\mu}{ }_{\nu}\right) h-E_{X} h^{\mu}{ }_{\nu}-\frac{1}{2} B^{\mu}{ }_{\nu}{ }^{\alpha \beta} k_{\alpha \beta} \\
& -\left(\nabla_{\alpha} T^{\mu}{ }_{\nu}-\frac{1}{2} B^{\mu}{ }_{\nu}{ }^{\sigma \beta} \nabla_{\alpha} f_{\sigma \beta}\right) \xi^{\alpha} \\
& +\left(2 T^{\alpha(\mu} g^{\beta}{ }_{\nu)}-B^{\mu}{ }_{\nu}{ }^{\sigma \alpha} f^{\beta}{ }_{\sigma}-A^{\mu}{ }_{\nu}{ }^{\alpha \beta}-T^{\mu}{ }_{\nu} g^{\alpha \beta}\right) \nabla_{\alpha} \xi_{\beta}
\end{aligned}
$$

where we have defined $k_{\mu \nu} \equiv \delta_{E} f_{\mu \nu}$.

We find the perturbed fluid variables by assuming the unperturbed tensor $f_{\mu \nu}$ is homogenous, i.e. $\bar{\nabla}_{\alpha} f_{\mu \nu}=0$, as chosen in [33], recalling the definition of "space" differentiation $\bar{\nabla}_{\mu} \psi \equiv \gamma_{\mu}^{\nu} \nabla_{\nu} \psi$ from (2.8)

$$
\begin{aligned}
& \delta \rho=-\left(B_{X}+\rho\right)\left(\frac{1}{2} h+\bar{\nabla}^{\alpha} m_{\alpha}\right)-\frac{1}{2}\left(B_{Y} \gamma^{\alpha \beta}+A_{Y} u^{\alpha} u^{\beta}\right) k_{\alpha \beta} \\
& +\left[\dot{\rho}+\frac{1}{2} \dot{f}^{\alpha \beta}\left(B_{Y} \gamma_{\alpha \beta}+A_{Y} u_{\alpha} u_{\beta}\right)+B_{Y} f^{\alpha \beta} K_{\alpha \beta}+\left(\rho+B_{X}\right) K\right] \chi \\
& -\left(A_{X}+A_{Y} f^{\beta}{ }_{\sigma} u^{\sigma} u_{\beta}+\rho\right) \dot{\chi}-f^{\alpha \beta} B_{Y} \bar{\nabla}_{\alpha} m_{\beta}, \\
& (\rho+P) v^{\lambda}=\left(\rho-C_{X}\right) \dot{\bar{m}}^{\lambda}-\left(P+C_{X}-C_{Y} u^{\sigma} u_{\beta} f_{\sigma}^{\beta}\right) \bar{\nabla}^{\lambda} \chi \\
& -C_{Y}\left\{\gamma^{\lambda(\alpha} u^{\beta)} k_{\alpha \beta}+\gamma^{\lambda \sigma} f_{\sigma}^{\beta} \dot{m}_{\beta}-m_{\beta} f^{\beta \sigma} K_{\sigma}^{\lambda}\right\} \text {, } \\
& \delta P=-\frac{1}{3}\left(3 D_{X}+P+2 E_{X}\right)\left(\frac{1}{2} h+\bar{\nabla}^{\beta} m_{\beta}\right)-\left(\frac{1}{2} B_{Y} u^{\alpha} u^{\beta}+\frac{1}{6}\left(3 D_{Y}+2 E_{Y}\right) \gamma^{\alpha \beta}\right) k_{\alpha \beta} \\
& -f^{\alpha \beta} \frac{1}{3}\left(3 D_{Y}+2 E_{Y}\right) \bar{\nabla}_{\alpha} m_{\beta}+\left(P-B_{X}+B_{Y} f^{\alpha \beta} u_{\alpha} u_{\beta}\right) \dot{\chi} \\
& +\left[\dot{P}+\frac{1}{3}\left(P+3 D_{X}+2 E_{X}\right) K+\frac{1}{3}\left(3 D_{Y}+2 E_{Y}\right) f^{\alpha \beta} K_{\alpha \beta}\right. \\
& \left.-\frac{1}{2} \dot{f}^{\alpha \beta}\left(\frac{1}{3}\left(3 D_{Y}+2 E_{Y}\right) \gamma_{\alpha \beta}+A_{Y} u_{\alpha} u_{\beta}\right)\right] \chi, \\
& P \Pi_{\lambda}^{\rho}=2\left(P-2 E_{X}\right)\left[\frac{1}{2} h_{\lambda}^{\rho}+\bar{\nabla}^{(\rho} m_{\lambda)}-\frac{1}{3} \gamma^{\rho}{ }_{\lambda}\left(\frac{1}{2} h+\bar{\nabla}_{\alpha} m^{\alpha}\right)\right] \\
& -E_{Y}\left[\left(\gamma^{\rho(\alpha} \gamma_{\lambda}^{\beta)}-\frac{1}{3} \gamma_{\lambda}^{\rho} \gamma^{\alpha \beta}\right) k_{\alpha \beta}+2 f^{\alpha \beta}\left(\gamma_{\alpha}{ }^{(\rho} \bar{\nabla}_{\lambda)} m_{\beta}--\frac{1}{3} \gamma_{\lambda}^{\rho} \bar{\nabla}_{\alpha} m_{\beta}\right)\right] .
\end{aligned}
$$

Assuming time reparameterisation invariance gives

$$
\begin{aligned}
\delta \rho & =-\left(A_{X}+\rho\right)\left(\frac{1}{2} h+\bar{\nabla}^{\alpha} \xi_{\alpha}\right)-\frac{1}{2}\left(B_{Y} \gamma^{\alpha \beta}+A_{Y} u^{\alpha} u^{\beta}\right) k_{\alpha \beta}-B_{Y} f^{\alpha \beta} \bar{\nabla}_{\alpha} \xi_{\beta}, \\
(\rho+P) v^{\lambda} & =\left(\rho-C_{X}\right) \dot{\xi}^{\lambda}-C_{Y}\left[\gamma^{\lambda(\alpha} u^{\beta)} k_{\alpha \beta}+f^{\alpha \beta}\left(\gamma_{\alpha}^{\lambda} \dot{\xi}_{\beta}-K_{\alpha}^{\lambda} \xi_{\beta}\right)\right]
\end{aligned}
$$




$$
\begin{aligned}
& \delta P=-\frac{1}{3}\left(3 D_{X}+P+2 E_{X}\right)\left(\frac{1}{2} h+\bar{\nabla}^{\beta} \xi_{\beta}\right)-\left(\frac{1}{2} B_{Y} u^{\alpha} u^{\beta}+\frac{1}{6}\left(3 D_{Y}+2 E_{Y}\right) \gamma^{\alpha \beta}\right) k_{\alpha \beta} \\
& -\frac{1}{3} f^{\alpha \beta}\left(3 D_{Y}+2 E_{Y}\right) \bar{\nabla}_{\alpha} \xi_{\beta} \\
& P \Pi_{\lambda}^{\rho}=2\left(P-2 E_{X}\right)\left[\frac{1}{2} h_{\lambda}^{\rho}+\bar{\nabla}^{(\rho} \xi_{\lambda)}-\frac{1}{3} \gamma_{\lambda}^{\rho}\left(\frac{1}{2} h+\bar{\nabla}_{\alpha} \xi^{\alpha}\right)\right] \\
& -E_{Y}\left\{\left(\gamma^{\rho(\alpha} \gamma_{\lambda}^{\beta)}-\frac{1}{3} \gamma_{\lambda}^{\rho} \gamma^{\alpha \beta}\right) k_{\alpha \beta}+2 f^{\alpha \beta}\left(\gamma_{\alpha}{ }^{(\rho} \bar{\nabla}_{\lambda)} \xi_{\beta}-\frac{1}{3} \gamma_{\lambda}^{\rho} \bar{\nabla}_{\alpha} \xi_{\beta}\right)\right\},
\end{aligned}
$$

and we obtain evolution equations for $P$ and $\rho$

$$
\begin{aligned}
& \dot{\rho}=-\frac{1}{2} \dot{f}^{\alpha \beta}\left(B_{Y} \gamma_{\alpha \beta}+A_{Y} u_{\alpha} u_{\beta}\right)-B_{Y} f^{\alpha \beta} K_{\alpha \beta}-\left(\rho+B_{X}\right) K \\
& \dot{P}=\frac{1}{3}\left(3 D_{Y}+2 E_{Y}\right) f^{\alpha \beta} K_{\alpha \beta}+\frac{1}{2}\left(D_{Y} \gamma_{\alpha \beta}-B_{Y} u_{\alpha} u_{\beta}\right) \dot{f}^{\alpha \beta}-\frac{1}{3}\left(P+3 D_{X}+2 E_{X}\right) K .
\end{aligned}
$$

\section{$5 \quad$ No preferred direction in the coupling tensors}

\subsection{Preferred direction}

Using $u_{\mu}$ and $\gamma_{\mu \nu}$ in the decomposition of the coupling tensors means that we have chosen a preferred direction for the Lagrangian. If we assume there is no preferred direction and only the full metric is seen in the coupling tensors, then (4.3) becomes

$$
\begin{aligned}
& A^{\mu \nu \alpha \beta}=A_{A} g^{\mu \nu} g^{\alpha \beta}+2 B_{A} g^{\alpha(\mu} g^{\nu) \beta}, \\
& B^{\mu \nu \alpha \beta}=A_{B} g^{\mu \nu} g^{\alpha \beta}+2 B_{B} g^{\alpha(\mu} g^{\nu) \beta}, \\
& C^{\mu \nu \alpha \beta}=A_{C} g^{\mu \nu} g^{\alpha \beta}+2 B_{C} g^{\alpha(\mu} g^{\nu) \beta} .
\end{aligned}
$$

In order to obtain (5.1a) we must set the coefficients in (4.3) as follows

$$
\begin{array}{lll}
A_{X}=A_{A}+2 B_{A} & A_{Y}=A_{B}+2 B_{B} & A_{Z}=A_{C}+2 B_{C} \\
B_{X}=-A_{A} & B_{Y}=-A_{B} & B_{Z}=-A_{C} \\
C_{X}=-B_{A} & C_{Y}=-B_{B} & C_{Z}=-B_{C} \\
D_{X}=A_{A} & D_{Y}=A_{B} & D_{Z}=A_{C} \\
E_{X}=B_{A} & E_{Y}=B_{B} & E_{Z}=B_{C} \\
& F_{Y}=-A_{B} . &
\end{array}
$$

\subsection{The perturbed fluid variables}

When we use the coupling tensors (5.2), then we find that the perturbed fluid variables when $\chi$ is decoupled are

$$
\delta \rho=\left(A_{A}-\rho\right)\left(\frac{1}{2} h+\bar{\nabla}_{\alpha} \xi^{\alpha}\right)+A_{B}\left(f^{\alpha \beta} \nabla_{\alpha} \xi_{\beta}+\frac{1}{2} k\right)-\frac{1}{3} B_{B} k^{\alpha \beta} u_{\alpha} u_{\beta}
$$




$$
\begin{aligned}
& (\rho+P) v^{\sigma}=B_{B}\left[u^{\nu} \gamma_{\mu}^{\sigma} k^{\mu}{ }_{\nu}+B_{B}\left(\gamma_{\mu}^{\sigma} f^{\mu \beta} \dot{\xi}_{\beta}-f^{\alpha \beta} K^{\sigma}{ }_{\alpha} \xi_{\beta}\right)\right]+\left(B_{A}+\rho\right) \dot{\xi}^{\sigma}, \\
& \delta P=-\left(\frac{1}{3}\left(2 B_{A}+P+3 A_{A}\right)\left(\frac{1}{2} h+\bar{\nabla}_{\alpha} \xi^{\alpha}\right)+\frac{1}{2} A_{B} k+\frac{1}{3} B_{B} \gamma^{\nu}{ }_{\mu} k^{\mu}{ }_{\nu}\right) \\
& -\frac{1}{3}\left(3 A_{B}+2 B_{B}\right) f^{\alpha \beta} \bar{\nabla}_{\alpha} \xi_{\beta} \\
& P \Pi_{\lambda}^{\rho}=2\left(P-2 B_{A}\right)\left(\frac{1}{2} h_{\lambda}^{\rho}+\bar{\nabla}^{(\rho} \xi_{\lambda)}-\frac{1}{3} \gamma_{\lambda}{ }_{\lambda}\left(\frac{1}{2} h+\bar{\nabla}_{\alpha} \xi^{\alpha}\right)\right) \\
& -2 B_{B}\left[\gamma^{\rho}{ }_{\mu} \gamma_{\lambda}^{\nu} k_{\nu}^{\mu}-\frac{1}{3} \gamma_{\mu}^{\nu} k^{\mu}{ }_{\nu} \gamma_{\lambda}^{\rho}+2 f^{\alpha \beta}\left(\gamma_{\alpha(\lambda} \bar{\nabla}^{\rho)} \xi_{\beta}-\frac{1}{3} \gamma_{\lambda}{ }_{\lambda} \bar{\nabla}_{\alpha} \xi_{\beta}\right)\right],
\end{aligned}
$$

and we can write the evolution equations (4.7) as

$$
\begin{aligned}
\dot{\rho} & =A_{B}\left(f^{\alpha \beta} K_{\alpha \beta}+\frac{1}{2} \dot{f}\right)-B_{B} \dot{f}^{\alpha \beta} u_{\alpha} u_{\beta}+\left(A_{A}-\rho\right) K \\
\dot{P} & =-\frac{1}{6}\left(2\left(P+3 A_{A}+2 B_{A}\right) K+3 A_{B}\left(\dot{f}+2 f^{\alpha \beta} K_{\alpha \beta}\right)+2 B_{B}\left(\dot{f}^{\alpha \beta} \gamma_{\alpha \beta}+2 f^{\alpha \beta} K_{\alpha \beta}\right)\right) .
\end{aligned}
$$

\section{Choosing a form for $f_{\mu \nu}$}

\subsection{Conformal and disformal choices}

An obvious choice for $f_{\mu \nu}$ is

$$
f_{\mu \nu}=A_{f} \phi g_{\mu \nu}+B_{f} \nabla_{\mu} \phi \nabla_{\nu} \phi
$$

where $A_{f}$ and $B_{f}$ are constants and $\phi$ is a scalar field. However, because this means that the variation of $f_{\mu \nu}$ contains the variation of the metric, we must modify (4.1). In fact, this choice means that (4.1) can be rewritten as the second variation of a Lagrangian of the form $\mathcal{L}_{(2)}\left(\delta_{O} L g_{\mu \nu}, \delta_{L} \phi, \nabla_{\mu} \delta_{L} \phi\right)$, i.e.

$$
\begin{aligned}
\mathcal{L}_{(2)}= & \mathcal{A}\left(\delta_{L} \phi\right)^{2}+\mathcal{B}^{\mu} \delta_{L} \phi \nabla_{\mu} \delta_{L} \phi+\frac{1}{2} \mathcal{C}^{\mu \nu} \nabla_{\mu} \delta_{L} \phi \nabla_{\nu} \delta_{L} \phi \\
& +\frac{1}{4}\left[\mathcal{Y}^{\alpha \mu \nu} \nabla_{\alpha} \delta_{L} \phi \delta_{L} g_{\mu \nu}+\mathcal{V}^{\mu \nu} \delta_{L} \phi \delta_{L} g_{\mu \nu}+\frac{1}{2} \mathcal{W}^{\mu \nu \alpha \beta} \delta_{L} g_{\mu \nu} \delta_{L} g_{\alpha \beta}\right],
\end{aligned}
$$

which was studied in [13]. The terms on the second line of (6.2) could be possibly be generated by "Beyond Horndeski" theories [34, 35]. The Beyond Horndeski theories contain terms in a specific combination to avoid the Ostrogradski instability, which would place constraints on the couplings $A, B^{\mu}$ and $C^{\mu \nu}$.

\subsection{Flat reference metric}

Another choice is a flat reference metric, i.e. $f_{\mu \nu}=\eta_{\mu \nu}$, although this choice does not simplify our perturbed fluid variables greatly while we are still using the generalised lagrangian (4.1). 


\section{Summary of results}

In this paper, we have

- summarised the calculations given in [14] for the perturbed fluid variables for a general dark Lagrangian of the form $\mathcal{L}_{\{2\}}=\mathcal{L}_{\{2\}}\left(\delta_{L} g_{\mu \nu}\right)$ by working in the synchronous gauge and in the perfectly elastic case, both with and without the imposition of time reparameterisation invariance

- obtained new constraints on the values of $w$ and $\mu$ for a realistic sound speed under various conditions, using data from the Planck satellite

- rewritten the perturbed fluid variables for general dark Lagrangians of the form $\mathcal{L}_{\{2\}}=\mathcal{L}_{\{2\}}\left(\delta_{L} \gamma_{\mu \nu}, \delta_{L} u_{\mu}\right), \mathcal{L}_{\{2\}}=\mathcal{L}_{\{2\}}\left(\delta_{L} u_{\mu}\right)$ and $\mathcal{L}_{\{2\}}=\mathcal{L}_{\{2\}}\left(\delta_{L} \gamma_{\mu \nu}\right)$ both in general and when time reparameterisation invariance is imposed

- obtained new evolution equations for $\dot{P}$ and $w$ for these new Lagrangians and repeated these calculations for tensor-metric theories where $\mathcal{L}_{\{2\}}=\mathcal{L}_{\{2\}}\left(\delta_{L} g_{\mu \nu}, \delta_{L} f_{\mu \nu}\right)$, using either a time-spatial metric split in the coupling tensors or using only the full metric

- found new evolution equations for $\rho$ and $P$ and rewritten the perturbed fluid variables in these theories

There are many different modified gravity theories which attempt to explain the accelerated expansion of the universe. By parameterising theories based on the second variation of the Lagrangian, we were able to develop a framework which can very quickly rule out various theories, and indeed we can rule out any theory which is purely a function of the variation of the time part of the metric. Our method could be used both in future models of massive gravity or to rule out large classes of theories when new observational results are found.

We have examined the connections between these theories and elastic dark energy. For our first case, the elastic dark energy framework can be straightforwardly used. We also placed constraints on the parameters of elastic dark energy using Planck results. Future work could examine whether other dark energy models can use this framework.

\section{Acknowledgments}

We would like to thank Richard Battye and David Burton for valuable discussions and suggestions, both on the content of this paper and the presentation.

\section{A Evolution of $w$}

We want to find an evolution equation for $w$, the equation of state parameter where $P=w \rho$. Using the conservation equation, (2.11) and (2.13), and defining

$$
\hat{\beta} \equiv \frac{\beta}{\rho},
$$


we obtain

$$
\dot{w}=3[w(1+w)-\hat{\beta}] H,
$$

which notably does not depend on $\mu$. Hence $w$ is constant if

$$
\hat{\beta}=w(1+w),
$$

and so

$$
w=-\frac{1}{2} \pm \frac{1}{2} \sqrt{1+4 \hat{\beta}}
$$

gives a stable universe. Using 2018 Planck data [28], the $68 \%$ constraint on $w$ is $w=$ $-1.028 \pm 0.032$ which in turn gives a constraint of $-0.004<\beta<0.106$.

\section{A.1 Sound speed}

The sound speed for elastic dark energy is given by [20]

$$
c_{s}^{2} \equiv \frac{\hat{\beta}+\frac{4}{3} \hat{\mu}}{1+w},
$$

where $\hat{\mu}=\frac{\mu}{\rho}$. Using (A.3), and in order that the sound speed fulfills $0 \leq c_{s}{ }^{2} \leq 1$, i.e. is real and sub-luminal, then for constant $w$ we must have

$$
-\frac{3}{4} w(1+w) \leq \hat{\mu} \leq \frac{3}{4}\left(1-w^{2}\right) .
$$

If we set $\hat{\mu}=0=\dot{w}$, then (A.6) gives that either $w=-1$ exactly, or

$$
0 \leq w \leq 1
$$

which leads to a contradiction with the acceleration of the universe, as acceleration requires $w<-\frac{1}{3}$. This means that either $w=-1$ or a stable universe with zero shear modulus cannot support acceleration of the universe and we therefore require a non-zero $\mu$. Using the 2018 Planck data [28] together with (A.6) gives us constraints of $-0.0477 \leq \hat{\mu} \leq 0.0599$. However, the assumption that we do not have a phantom equation of state, i.e. we require $w \geq-1$, gives $0 \leq \hat{\mu} \leq 0.0599$.

Open Access. This article is distributed under the terms of the Creative Commons Attribution License (CC-BY 4.0), which permits any use, distribution and reproduction in any medium, provided the original author(s) and source are credited.

\section{References}

[1] Supernova Cosmology Project collaboration, S. Perlmutter et al., Measurements of $\Omega$ and $\Lambda$ from 42 high redshift supernovae, Astrophys. J. 517 (1999) 565 [astro-ph/9812133] [INSPIRE].

[2] Supernova Search Team collaboration, A.G. Riess et al., Observational evidence from supernovae for an accelerating universe and a cosmological constant, Astron. J. 116 (1998) 1009 [astro-ph/9805201] [INSPIRE]. 
[3] C. Heymans et al., CFHTLenS: the Canada-France-Hawaii telescope lensing survey, Mon. Not. Roy. Astron. Soc. 427 (2012) 146 [arXiv:1210.0032] [InSPIRE].

[4] Planck collaboration, P.A.R. Ade et al., Planck 2013 results. XV. CMB power spectra and likelihood, Astron. Astrophys. 571 (2014) A15 [arXiv:1303.5075] [InSPIRE].

[5] Planck collaboration, P.A.R. Ade et al., Planck 2013 results. XVII. Gravitational lensing by large-scale structure, Astron. Astrophys. 571 (2014) A17 [arXiv:1303.5077] [InSPIRE].

[6] DES collaboration, T. Abbott et al., The dark energy survey, astro-ph/0510346 [INSPIRE].

[7] LSST Dark Energy Science collaboration, A. Abate et al., Large Synoptic Survey Telescope: dark energy science collaboration, arXiv:1211.0310 [INSPIRE].

[8] R.A. Battye, F. Pace and D. Trinh, Gravitational wave constraints on dark sector models, Phys. Rev. D 98 (2018) 023504 [arXiv: 1802.09447] [InSPIRE].

[9] T. Clifton, P.G. Ferreira, A. Padilla and C. Skordis, Modified gravity and cosmology, Phys. Rept. 513 (2012) 1 [arXiv:1106.2476] [INSPIRE].

[10] J.A. Pearson, Effective field theory for perturbations in dark energy and modified gravity, arXiv: 1205.3611 [INSPIRE].

[11] J. Bloomfield and J. Pearson, Simple implementation of general dark energy models, JCAP 03 (2014) 017 [arXiv: 1310.6033] [INSPIRE].

[12] R.A. Battye and J.A. Pearson, Massive gravity, the elasticity of space-time and perturbations in the dark sector, Phys. Rev. D 88 (2013) 084004 [arXiv:1301.5042] [INSPIRE].

[13] R.A. Battye and J.A. Pearson, Effective action approach to cosmological perturbations in dark energy and modified gravity, JCAP 07 (2012) 019 [arXiv: 1203.0398] [INSPIRE].

[14] R.A. Battye and J.A. Pearson, Computing model independent perturbations in dark energy and modified gravity, JCAP 03 (2014) 051 [arXiv:1311.6737] [INSPIRE].

[15] C. Skordis, Consistent cosmological modifications to the Einstein equations, Phys. Rev. D 79 (2009) 123527 [arXiv:0806.1238] [INSPIRE].

[16] T. Baker, P.G. Ferreira, C. Skordis and J. Zuntz, Towards a fully consistent parameterization of modified gravity, Phys. Rev. D 84 (2011) 124018 [arXiv:1107.0491] [INSPIRE].

[17] T. Baker, P.G. Ferreira and C. Skordis, The parameterized post-Friedmann framework for theories of modified gravity: concepts, formalism and examples, Phys. Rev. D 87 (2013) 024015 [arXiv:1209.2117] [INSPIRE].

[18] Virgo and LIGO ScIEnTIfIC collaborations, B. Abbott et al., GW170817: observation of gravitational waves from a binary neutron star inspiral, Phys. Rev. Lett. 119 (2017) 161101 [arXiv: 1710.05832] [INSPIRE].

[19] C. de Rham and S. Melville, Gravitational rainbows: LIGO and dark energy at its cutoff, arXiv: 1806.09417 [INSPIRE].

[20] J.A. Pearson, Material models of dark energy, Annalen Phys. 526 (2014) 318 [arXiv: 1403.1213] [INSPIRE].

[21] B. Carter, Interaction of gravitational waves with an elastic solid medium, in Les Houches Summer School on Gravitational Radiation, Les Houches, France, 2-21 June 1982 [gr-qc/0102113] [INSPIRE]. 
[22] B. Carter, Speed of sound in a high-pressure general-relativistic solid, Phys. Rev. D 7 (1973) 1590 [InSPIRE].

[23] B. Carter, Rheometric structure theory, convective differentiation and continuum electrodynamics, Proc. Roy. Soc. Lond. A 372 (1980) 169.

[24] J.L. Friedman and B.F. Schutz, On the stability of relativistic systems, Astrophys. J. 200 (1975) 204 [Erratum ibid. 222 (1978) 1119].

[25] B. Carter and H. Quintana, Foundations of general relativistic high-pressure elasticity theory, Proc. Roy. Soc. Lond. A 331 (1972) 57.

[26] O.J. Tattersall, M. Lagos and P.G. Ferreira, Covariant approach to parametrized cosmological perturbations, Phys. Rev. D 96 (2017) 064011 [arXiv:1706.10091] [INSPIRE].

[27] R.A. Battye, A. Moss and J.A. Pearson, Constraining dark sector perturbations I: cosmic shear and CMB lensing, JCAP 04 (2015) 048 [arXiv: 1409.4650] [INSPIRE].

[28] Planck collaboration, P.A.R. Ade et al., Planck 2015 results. XIII. Cosmological parameters, Astron. Astrophys. 594 (2016) A13 [arXiv: 1502.01589] [inSPIRE].

[29] R.A. Battye and A. Moss, Cosmological perturbations in elastic dark energy models, Phys. Rev. D 76 (2007) 023005 [astro-ph/0703744] [INSPIRE].

[30] W. Hu, U. Seljak, M.J. White and M. Zaldarriaga, A complete treatment of CMB anisotropies in a FRW universe, Phys. Rev. D 57 (1998) 3290 [astro-ph/9709066] [INSPIRE].

[31] C. de Rham, G. Gabadadze and A.J. Tolley, Resummation of massive gravity, Phys. Rev. Lett. 106 (2011) 231101 [arXiv:1011.1232] [InSPIRE].

[32] A. Schmidt-May and M. von Strauss, Recent developments in bimetric theory, J. Phys. A 49 (2016) 183001 [arXiv:1512.00021] [InSPIRE].

[33] M. Lagos and P.G. Ferreira, A general theory of linear cosmological perturbations: bimetric theories, JCAP 01 (2017) 047 [arXiv: 1610.00553] [INSPIRE].

[34] J. Gleyzes, D. Langlois, F. Piazza and F. Vernizzi, Healthy theories beyond Horndeski, Phys. Rev. Lett. 114 (2015) 211101 [arXiv: 1404.6495] [INSPIRE].

[35] J. Ben Achour, M. Crisostomi, K. Koyama, D. Langlois, K. Noui and G. Tasinato, Degenerate higher order scalar-tensor theories beyond Horndeski up to cubic order, JHEP 12 (2016) 100 [arXiv: 1608.08135] [INSPIRE]. 